\title{
QUEEN'S
UNIVERSITY
BELFAST
}

\section{Relationship between monetary delay discounting and obesity: a systematic review and meta-regression}

Tang, J., Chrzanowski-Smith, O. J., Hutchinson, G., Kee, F., \& Hunter, R. F. (2018). Relationship between monetary delay discounting and obesity: a systematic review and meta-regression. International Journal of Obesity. https://doi.org/10.1038/s41366-018-0265-0

Published in:

International Journal of Obesity

Document Version:

Peer reviewed version

Queen's University Belfast - Research Portal:

Link to publication record in Queen's University Belfast Research Portal

Publisher rights

(C) 2018 Springer Nature Limited.

This work is made available online in accordance with the publisher's policies. Please refer to any applicable terms of use of the publisher.

\section{General rights}

Copyright for the publications made accessible via the Queen's University Belfast Research Portal is retained by the author(s) and / or other copyright owners and it is a condition of accessing these publications that users recognise and abide by the legal requirements associated with these rights.

Take down policy

The Research Portal is Queen's institutional repository that provides access to Queen's research output. Every effort has been made to ensure that content in the Research Portal does not infringe any person's rights, or applicable UK laws. If you discover content in the Research Portal that you believe breaches copyright or violates any law, please contact openaccess@qub.ac.uk. 


\section{Title page}

Title:

Relationship between monetary delay discounting and obesity: A systematic review and meta-regression

Running title: Monetary Delay Discounting and Obesity

\section{Authors:}

Jianjun Tang ${ }^{1,2,}{ }^{*}$, Oliver Chrzanowski-Smith ${ }^{3}$, George Hutchinson ${ }^{2,4}$, Frank Kee ${ }^{2}$, Ruth F. Hunter ${ }^{2}$

\section{Affiliations:}

${ }^{1}$ School of Agricultural Economics and Rural Development, Renmin University of China, Beijing 100872, China

${ }^{2}$ Centre for Public Health/UKCRC Centre of Excellence for Public Health (NI), Queen's University Belfast, Belfast, Northern Ireland, UK

${ }^{3}$ Department for Health, University of Bath, Bath, UK

${ }^{4}$ Gibson Institute for Land, Food and Environment, School of Biological Sciences, Queen's University Belfast, Belfast, Northern Ireland, UK

Email addresses:

Jianjun Tang (j.tang@qub.ac.uk)

Oliver Chrzanowski-Smith (O.J.Chrzanowski-Smith@bath.ac.uk)

George Hutchinson (g.hutchinson@qub.ac.uk)

Frank Kee (f.kee@qub.ac.uk)

Ruth F. Hunter (ruth.hunter@qub.ac.uk)

\section{${ }^{*}$ Corresponding author:}

Dr. Jianjun Tang, School of Agricultural Economics and Rural Development, Renmin University of China, 59 Zhongguancun Ave., Beijing 100872, CHINA; E-mail: j.tang@qub.ac.uk; Tel: +86 (0)10-62511061.

Conflicts of interest: The authors have no conflicts of interest. 


\section{Relationship between monetary delay discounting and obesity: A} systematic review and meta-regression

Background and objectives: Previous studies have documented that high rates of delay discounting are associated with obesity. However, studies utilizing monetary reward experiments typically report no associations, as opposed to positive associations apparent in studies utilising food-reward experiments. Our objective was to investigate the reasons behind the mixed evidence from a methodological perspective using systematic review and meta-analytic methodologies.

Methods: Seven databases (EMBASE, MEDLINE, PsycINFO, Scopus, Web of Science, Econlit and IBSS) were systematically searched. Logistic meta-regression was applied to identify the determinants of a significant association and risk of bias was assessed using a modified form of the Newcastle Ottawa cohort scale.

Results: A total of 59 studies were identified, among which 29 studies (49.2\%) found a significant positive association and 29 (49.2\%) reported no association. A higher proportion of significant and positive associations was reported in those studies utilizing 'best-practice' methods (i.e. appropriate measurement models) to estimate monetary delay discounting $(15 / 27 ; 55.6 \%)$ and incentive-compatible experiments $(10 / 16 ; 62.5 \%)$ than those using non-'best-practice' methods $(14 / 34 ; 41.2 \%)$ and hypothetical experiments (19/43; 44.2\%). All five studies utilizing both 'best-practice' methods and incentive-compatible experiments generated a positive and significant relationship. Results from a logistic meta-regression also suggested that studies 
employing incentive-compatible experiments (OR: $4.38,95 \% \mathrm{Cl}=1.05-18.33, \mathrm{p}$-value: 0.04), 'best-practice' methods (OR: $4.40,95 \% \mathrm{Cl}=0.88-22.99, p$-value: 0.07 ), parametric methods (OR: $3.36,95 \% \mathrm{Cl}=0.83-13.57$, p-value: 0.04 ), those conducted in children/adolescent populations (OR: $3.90,95 \% \mathrm{Cl}=0.85-17.88, \mathrm{p}$-value: 0.08 ), and those with larger sample size (OR: $1.91,95 \% \mathrm{Cl}=1.15-3.18$, $\mathrm{p}$-value: 0.01 ) tended to show positive and significant associations between delay discounting and obesity.

Conclusions: This review suggests that the mixed evidence to date is a result of methodological heterogeneity, and that future studies should utilise 'best practice' methods.

Keywords: Systematic review; Obesity; Delay discounting; Financial incentives; Present-bias; Incentive-compatible

\section{Introduction}

Obesity is a major global risk factor for morbidity and mortality [1] and entails substantial economic costs [2]. In 2013-2014, 35\% of adult men and 40\% adult women in the United States were obese (body mass index $\geq 30$ ) [3], a figure forecasted to increase to over $50 \%$ by 2030 [4]. Accordingly, obesity represents a major current and future public health challenge. To date, traditional interventions to promote healthy dietary patterns have had modest success in reducing these trends, with weight loss maintenance rare and weight regain common [5]. Thus, there is a recognized need to explore new obesity reduction and prevention strategies [6]. 
While in its infancy, behavioural economics has provided new insights and sparked interest among public health researchers and the United States government $[7,8]$. An increasingly popular strategy is the provision of financial incentives to aid weight-loss and encourage physical activity and dietary-related behavioral change $[9,10]$. The principle behind financial incentives is to capitalize upon the notion of delay discounting, i.e. the tendency for an individual to place greater value on the present benefits of an action relative to the future benefits [7].

Delay discounting, also commonly known as discount rate, time preference, temporal discounting or time discounting, is typically elicited through a binary question (e.g. Would you choose $\$ 100$ today or $\$ 150$ one year from today?"), or series of binary questions, that ask an individual to choose between an immediate, smaller reward or a delayed, larger reward [11]. Individuals who choose the smaller, immediate reward are said to have a high discount rate and thus, are less likely to resist the temptation for an immediate gratification which might harm their future health [7].

There is rich literature on the role of delay discounting in shaping our health behaviours. Early research focused heavily on addictive health behaviours, where systematic reviews report positive associations between delay discounting and both smoking and drug use $[12,13]$. Considering the alarming prevalence over recent years, a growing body of research has shifted attention towards unhealthy diets as well as obesity. Individuals with higher discount rates are assumed to be less likely to resist tempting yet unhealthy foods and eventually become obese. Barlow et al. concluded that there was consistent evidence of an association between delay discounting and obesity, i.e. a higher delay discounting is associated with an increased weight [14]. 
Interestingly, this was dependent upon the method used to elicit delay discounting: studies utilizing hypothetical and monetary reward choice tasks typically reported no associations as opposed to positive associations apparent in studies using food choice tasks. In contrast, a systematic review by Story et al. [15], albeit focusing on a range of health behaviours, reported strong evidence of an association between monetary elicited temporal discounting and unhealthy behaviours, noting an emerging relationship with obesity. This mixed finding, where obesity was commonly associated with delay discounting elicited from food choice tasks but not with monetary-based methods, requires further exploration.

We hypothesize that this is a result of the largely heterogeneous methods used to elicit monetary delay discounting. For example, various studies have either unjustifiably assumed a parametric form of discount function or used hypothetical rather than real rewards in their choice tasks, possibly biasing their delay discounting estimates. Various studies have employed the Mazur-hyperbolic functional form [16], which does not necessarily characterise individual discount behaviours and other flexible hyperbolic discount functions have rarely been adopted [17]. Further, a valuable and more valid technique is to use real rather than hypothetical rewards when possible, because real monetary rewards are familiar, fungible and have potential to deal with hypothetical bias, and thus likely eliciting more reflective discount rates [18]. Thus, the null findings reported with monetary delay discounting by Barlow et al. [14], as noted by the authors themselves, should be interpreted with caution as only two out of the 41 included studies used incentive-compatible designs, i.e. real monies were actually paid to participants depending on their chosen 
immediate or future rewards. Further, methodological heterogeneity was not taken into consideration.

Therefore, in light of the above issues, the aim of the current systematic review was to investigate whether the previously reported null association between obesity and monetary delay discounting may be explained by methodological heterogeneity, with a particular focus on inter alia whether the studies utilized incentive-compatible experiments and 'best-practice' methods, i.e. appropriate methods to estimate monetary delay discounting. Using systematic review, meta-regression and risk of bias methodologies, evidence was synthesized both numerically and empirically to draw robust conclusions.

\section{Methods}

\section{Search Strategy and Eligibility Criteria}

The protocol is registered on PROSPERO (registration number CRD42015026786). Seven electronic databases (EconLit, EMBASE, International Bibliography of the Social Sciences, Medline, PsycINFO, Scopus and Web of Science) were searched for studies published since inception to May 2017. A combination of key terms relating to 'delay discounting' and 'obesity' was searched (Supplementary Information S1). Inclusion criteria were:

i. Used a monetary measure of 'delay discounting'

ii. Reported a measure of 'obesity' (i.e. body mass index; body fat percentage)

iii. Tested for a direct association between 'delay discounting' and 'obesity' 
iv. Involved a non-clinical (i.e. no current clinically diagnosed condition or disease) population

v. Retrospective or prospective cohort, cross-sectional study design or randomized control trial $(\mathrm{RCT})$

115 vi. English language

116 vii. Full-text available

117 RCTs were included only if the association between delay discounting and obesity was 118 analyzed at baseline to prevent any confounding effects of the intervention on the 119 association. Studies that did not report the health status of participants were assumed 120 to be non-clinical and were included. Where studies also involved a clinical population 121 group, the data utilized in the paper was only that which tested the association for a 122 non-clinical population group. All age ranges were included. Studies that used non123 monetary measures of delay discounting were excluded.

124 Identified records were screened against the eligibility criteria independently by two 125 researchers $(X X)$ in two phases: (1) titles and abstracts; then, (2) full texts. Any 126 disagreements were resolved by discussion between members of the research team.

127 Reference lists of included studies were manually searched for further eligible papers.

\section{Data Extraction}

129 Data was extracted by one reviewer (XX) and then independently cross checked by 130 two other reviewers (XX and XX). Key study characteristics and outcome(s) of interest 131 were extracted and tabulated for each study including sample size, population group, 132 measure of delay discounting and obesity, primary statistical analysis conducted for 
delay discounting-obesity association, results ( $p$-values, standard errors, and $95 \%$ confidence intervals) and summary/main finding(s).

\section{Evidence Synthesis}

In studies that measured both monetary and non-monetary measures of delay discounting, only data that used monetary elicited delay discounting were extracted and analyzed. Methods to elicit an individual's monetary delay discounting were classified and ranked according to whether discount rates were estimated by 'best practice' methods and whether real or hypothetical payments were used. The methods were described as 'best practice' if: (i) either the non-parametric method, Area Under Curve (AUC), or, the parametric methods assuming dual-parameter discount functions, e.g. Quasi-hyperbolic, Fixed-cost hyperbolic, Generalizedhyperbolic, and Saturating-hyperbolic) and (ii) incentive-compatible tasks when respondents had chances to be paid for real, depending on their own choices to these tasks (Supplementary Information S2). Studies that referred to a group as 'normal weight' or control group are referred to as 'healthy weight'. A statistically significant delay discounting-obesity association was defined as a $p$-value less than 0.05 . To quantitatively identify the determinants of a significant association, a logistic metaregression analysis using STATA was conducted (version 13.0; Stata Corp., College Station, TX, USA). To test the robustness of the meta-regression results, a backward stepwise procedure was followed such that the insignificant predictor variables in the initial model were deleted one by one starting from the factor with the highest $p$-value until the $p$-values of all remaining predictor variables were below 0.10 (final model) 
155

156

157

158

159

160

161

162

163

164

165

166

167

168

169

170

171

172

173

174

175

[19]. However, as the delay discounting estimates had different metrics resulting from the heterogeneous methods, a meta-analysis was not appropriate.

\section{Assessment of risk of bias}

Risks of bias were assessed independently by two reviewers ( $X X$ and $X X)$ using a modified form of the Newcastle Ottawa cohort scale for cross-sectional studies [20] (Supplementary Information S3).

\section{Results}

The PRISMA flow diagram [21] depicted in Figure 1 shows the results of the literature search. In summary, 1005 records were identified following database searching and 59 studies were included in the review. Study characteristics and main findings are reported in Table 1. Large heterogeneity existed among the studies, especially for the type of monetary tasks used to elicit delay discounting, the statistical analysis applied and sample sizes (ranging from 28 [22] to 42,863 [23] participants). The majority of studies were conducted in the USA $(n=34)$, followed by Europe $(n=16)$, Asia $(n=6)$, Canada $(n=1)$, Australia $(n=1)$ and multiple countries $(n=1)$. Fifty-eight studies were based on cross-sectional analysis of the association between delay discounting and obesity, and one was a longitudinal study [24]. Forty-seven (79.7\%) studies were conducted in adults and twelve (20.3\%) in children.

Overall the quality of statistical tests and reporting tended to be high with a mean score of 5.03 (S.D. 1.47, range $=1-8)$. Ten (16.9\%) studies were classified as 'high' quality (more than 6 stars), forty (67.8\%) 'medium' quality (4-6 stars) and nine (15.3\%) 
'low' quality (less than 4 stars). For the complete risk of bias analysis conducted using the Newcastle-Ottawa Scale, see Supplementary Information S3 and S4.

\section{Heterogeneity of methods in the elicitation of monetary delay discounting}

A range of different methods was used to elicit monetary delay discounting (Supplementary Information S2) and to analyze its association with obesity. Only a small proportion of studies $(16 / 59 ; 27 \%)$ made their inter-temporal choice tasks incentive-compatible so that participants had a chance to be paid (often) for one randomly determined choice they made [25-38].

Two studies $[39,40]$ conducted both parametric and non-parametric analysis taking the total number of analyzes to 61 . Among the 25 parametric studies, 2 studies elicited delay discounting through Exponential discounting $[25,41]$ and 23 utilized hyperbolic discounting (Mazur-hyperbolic, $n=18$, [26, 28, 31, 39, 40, 42-52]; Quasi-hyperbolic, $n=2,[34,53]$; Generalized-hyperbolic, $n=1,[54]$; Saturating-hyperbolic, $n=1$, [17]; and Fixed-cost hyperbolic, $n=1,[32])$. Among the 36 non-parametric studies, 21 studies utilized the Area Under Curve (AUC) method [22, 29, 30, 36, 39, 40, 55-69] and 15 used proxy measures $[23,24,27,33,35,37,38,70-77]$. Among those using proxy measures, five studies were based on a single multiple-choice trade-off question $[23,24,27,72$, 77], five used indifference points $[33,71,73,75,76]$, two used an open-ended future equivalent question $[35,74]$, and three used the number of patient choices $[37,38$, 70].

\section{Overall association}


Among the 59 included studies, 29 studies (49\%) found a significant positive association between delay discounting and obesity, 29 (49\%) reported no association and $1(2 \%)$ found a significant negative association (Table 1 ).

[Insert Table 1 about here]

\section{'best-practice' vs. non-'best-practice' analysis}

Studies utilizing 'best-practice' methods tended to reveal a higher proportion of significant associations than those using non-'best-practice' methods (Table 1). Among the 27 studies utilizing 'best-practice' methods, 15 (55.6\%) studies produced a significant positive association and 12 (44.4\%) reported no association. In comparison, non-'best-practice' methods resulted in a lower proportion of positive associations $(14 / 34 ; 41.2 \%)$, a higher proportion with no association $(19 / 34 ; 55.9 \%)$, and one $(2.9 \%)$ significant negative association.

\section{Parametric Mazur-hyperbolic discounting vs. non-parametric AUC}

Mazur-hyperbolic discounting is the main parametric method used whereas AUC is the main non-parametrical method. Among the 18 studies that applied the Mazurhyperbolic discount function, eight (44.4\%) produced a significant positive association and 10 (55.6\%) found no association. In the 21 studies that used the AUC method, a significant positive association was evident in nine $(42.9 \%)$ and no association was apparent in 12 (57.1\%) studies. Two studies reported results from both the Mazurhyperbolic and non-parametric AUC methods and found no association between delay discounting and obesity, irrespective of the method used $[39,40]$. 
Among the 16 studies that used real monetary rewards, ten (62.5\%) found a significant positive association between delay discounting and obesity, five reported no association (31.2\%) and one (6.3\%) produced a significant negative association. In contrast, 19 (44.2\%) out of the 43 studies that used hypothetical monetary rewards

225 found an overall significant positive association between delay discounting and obesity, and 24 (55.8\%) reported no association.

In summary, from the above a trend is observed to support the conjecture that either 'best-practice' methods or real rewards resulted in a somewhat higher proportion of positive and significant associations between obesity and delay discounting than their counterpart non-'best-practice' or hypothetical rewards analysis. Further, it is noteworthy that the five real reward studies employing 'best-practice' methods exceptionally generated a positive and significant relationship [29, 30, 32, 34, 36]. If the five real reward studies using Mazur-hyperbolic $[26,28,31]$ are also included, the same trend holds for 9 out of 10 studies. Conversely, it should be noted that a 'bestpractice' method or a real reward design alone does not lead to many significant and positive results. Studies utilizing 'best-practice' methods but hypothetical rewards produced positive and significant relationships in only 10 (45.5\%) out of 22 studies. In a similar vein, the real rewards studies that did not adopt a 'best-practice' approach (e.g. used Proxy and Exponential etc.) produced significant results in 5 (45.5\%) out of 11 studies. Thus, 'best-practice' methods and incentive-compatible environments seem to be the two necessary conditions for a positive and significant relationship.

\section{Objective vs. subjective measures of obesity}


All included studies assessed obesity through BMI. Twelve studies (20.3\%) did not state whether their measures of BMI were objectively or subjectively reported. Among the 29 studies that have used objectively measured BMI, 12 (41.4\%) reported a significant positive association and 17 (58.6\%) found no association. By contrast, 12 (66.7\%) out of 18 studies with self-reported BMI found a significant positive association and $6(33.3 \%)$ reported no association.

\section{Quantitative analysis}

The logistic meta-regression model (Table 2) shows that none of the factors were robust and significant predictors of a significant and positive association in both initial and final models, albeit those studies employing incentive-compatible experiments $(\mathrm{OR}=3.86,95 \% \mathrm{Cl}=0.81,18.43 ; \mathrm{OR}=4.38,95 \% \mathrm{Cl}=1.05,18.33)$, 'best-practice' methods $(\mathrm{OR}=6.54,95 \% \mathrm{Cl}=0.87,49.28 ; \mathrm{OR}=4.40,95 \% \mathrm{Cl}=0.88,22.99)$, parametric methods $(\mathrm{OR}=5.38,95 \% \mathrm{Cl}=1.01,28.73 ; \mathrm{OR}=3.36,95 \% \mathrm{Cl}=0.83,13.57)$, those conducted in children/adolescent populations $(\mathrm{OR}=4.54,95 \% \mathrm{Cl}=0.85,24.34 ; \mathrm{OR}=$ $3.90,95 \% \mathrm{Cl}=0.85,17.88)$, and those with larger sample size $(\mathrm{OR}=1.67,95 \% \mathrm{Cl}=0.89$, 3.13; $\mathrm{OR}=1.91,95 \% \mathrm{Cl}=1.15,3.18)$ tended to reveal a positive significant association, echoing the earlier finding that 'best-practice' methods or real rewards alone do not lead to many significant and positive results. However, when ten "low" quality studies were excluded from the analysis, those studies utilizing 'best-practice' methods and parametric methods are more likely to report a positive and significant association (Supplementary Information S5). 


\section{Methodological moderators}

The association between delay discounting and obesity was not moderated by specific trial/time delay, reward size and weight status threshold used (Supplementary Information S6).

\section{Discussion}

In summary, this study demonstrates that monetary elicited delay discounting was only associated with obesity in the studies in which incentive-compatible experiments were used and 'best-practice' methods to estimate delay discounting were employed.

Looking broadly at the associations found in all included studies, an equal number of studies found positive or no associations between monetary-elicited delay discounting

277 and obesity. This is consistent with the overall conclusion of Barlow et al. [14] who stated that there was a lack of evidence that a monetary-elicited delay discounting was associated with obesity. This lack of clear association is not aligned with the predictions of theoretical concepts of delay discounting and the prevailing hypothesis

281 commonly held by many researchers from the field $[11,78]$. Nor is it in line with the fact that delay discounting elicited from food-based tasks strongly relate to consuming unhealthy foods and obesity as observed by Barlow et al. However, the trend becomes much clearer if the studies are rated according to methodological appropriateness and incentive-compatibility. We found that studies where real rewards were at stake and 
reported positive and significant relationships. Furthermore, these two conditions are complementary but seem not to act alone (i.e. studies adopting 'best-practice' methods or real reward alone result in fewer identified associations). Thus we conjecture that the broadly null relationship between monetary delay discounting and obesity reported by Barlow et al. is conflated by critical methodological issues. We suggest that findings should be interpreted with caution in studies which fail to adopt 293 the 'best-practice' methods, or which use hypothetical choice tasks.

Considerable heterogeneity was evident across all of the included studies on the basis of various methodological issues, echoing previous discussions in the literature [14, $52,61]$. Regarding whether the method applied was parametric or non-parametric, the present results revealed that the former method tends to reveal a higher parametric methods. The parametric Mazur-hyperbolic discount function and non- 
The basic advantage over the typically-modelled Mazur-hyperbolic function of the more flexible hyperbolic functional forms, e.g. Fixed-cost hyperbolic and Quasihyperbolic etc., is that they incorporate two parameters each of which represents the two elements of delay discounting: 1) a conventional discount rate which refers to the extent to which future rewards are discounted; and (2) time-inconsistency which means the relative discount rate between two proximal delays is higher than the relative discount rate between two distal delays. It is intriguing that advances in neuroeconomics have provided novel insights into the neural mechanisms associated with the discounting of delayed rewards where two distinguishable brain regions are involved, with one associated with time-inconsistency (also referring to present-bias, i.e. sensitivity to the immediacy of rewards irrespective of reward magnitude) and the other related to discount rate (the long-run delay of the absolute magnitude of rewards irrespective of delay immediacy) $[79,80]$. In addition, one should be aware that present-bias is not universally observed in all subjects, while discounting is an inherent phenomenon (detailed discussions see Andersen et al. [18]). Six of the included studies conducted analyses relating to the two elements, although not all applied the parametric methods per se $[27,32,34,53,54,73]$. Three studies found

327 that a higher discount rate and greater present-bias were both significantly associated with higher BMI, respectively [17, 53, 54]. By contrast, Simmank et al. [34] found that

329 only discount rate but not present bias was related to BMI. Instead of a parametric estimation, Ikeda et al. [73] constructed a dummy for present-bias and again found that only discount rate (not present bias) was related to BMI. As mentioned earlier, very few studies took account of the two related yet distinct elements of delay discounting. We conjecture that the majority of the included parametric studies 
assuming a Mazur-hyperbolic function and the non-parametric AUC method failed to disentangle the two differentiated elements, resulting in pseudo non-associations between monetary delay discounting and obesity.

Strengths of this study included the addition of numerous studies with hypothetical monetary tasks, and with delay discounting elicited from real-monetary rewards, which facilitated investigation of why a monetary-elicited delay discounting was not associated with obesity. To our knowledge, this is the first systematic review that has attempted to elucidate the methodological implications.

\section{Implications for public health research and practice}

In times of economic hardship and spiraling healthcare costs, return on investment is a high priority for governments, especially with regards to the potential for interventions to change behavior that use financial rewards. With the concern that policy makers might 'jump the gun' by implementing interventions before convincing evidence exists $[7,81]$, studies based on 'best-practice' will help direct and develop future delay discounting and obesity research to ensure studies are methodologically robust. This will allow more definitive conclusions to be drawn, helping policy makers to make balanced, evidence-based decisions. The decomposition of delay discounting into its two components suggests that incentive strategies derived from each of the two elements differ in that the discount rate determines the overall level of incentives whereas the time-inconstancy factor determines whether incentives should be changed to match the decreasing discount rates over time.

\section{Future directions}


Firstly, there is a need for future research to validate the conditions for eliciting monetary delay discounting, with regards to both the real rewards utilized and the 'best-practice' methods applied. This will allow stronger and more comparable study designs, which will ultimately provide greater clarity on the association between delay discounting and obesity. As highlighted by Barlow et al. [14] and Story et al. [15], there is a need for longitudinal study designs and to control for risk aversion. All but one study included in the current systematic review was cross-sectional, preventing any information on causality, if a potential association exists. Few studies controlled for risk aversion, now considered to avoid upward-biases to the discount rate estimates [18]. Secondly, given the fact that financial incentives are increasingly used in weight loss intervention programs, how to design incentive levels to match the two related yet distinct underlying processes of delay discounting, i.e. level of discounting and time-inconsistency, are open questions for future research. This may have important implications for the applicability of delay discounting in weight loss interventions, especially when incentives are contingent on achieving a future weight loss target.

371 Lastly, a previously overlooked area is to test for demographic differences in the 372 association between delay discounting and obesity. Among the very few studies to 373 date which investigated this, the majority found no evidence of moderation effects 374 from demographic factors such as gender, age. While this finding should be 375 interpreted with caution due to the small numbers of studies, the potential 376 demographic moderators are highly relevant for the incorporation of delay 377 discounting into the design and implementation of obesity strategies, highlighting the need to consider them in future research. 


\section{Conclusions}

380 In research conducted to date on the association between monetary-elicited delay

381 discounting and obesity, there have been an equal number of significant positive and

382 insignificant positive associations. This study revealed that only the studies adopting

383 'best-practices' and incentive-compatible tasks have reported a significant association

384 between delay discounting and obesity. This conclusion may be relevant to studies

385 which link delay discounting to other real-world health behaviors in general. Given the

386 heterogeneous methods adopted in the literature, the current evidence suggests a

387 need for future studies to utilize 'best-practice' methods and incentive-compatible

388 designs to more validly discern the association between monetary delay discounting 389 and obesity, allowing policy makers to draw definitive conclusions based on 390 methodologically robust evidence. Prior to the implementation of behavioral change 391 programs, the investigation of delay discounting of the target population and the 392 decomposition into its two components, i.e. discount rate and time-inconsistency, 393 could provide insights into designing incentive strategies to improve effectiveness of 394 these programs.

The authors declare no conflict of interest. 
400

401

402

403

404

405

406

407

408

409

410

411

412

413

414

415

416

$417 \quad 2011 ; 378(9793): 815-25$.

418 5. Dombrowski SU, Knittle K, Avenell A, Araujo-Soares V, Sniehotta FF. Long term

419 maintenance of weight loss with non-surgical interventions in obese adults: Systematic

420 review and meta-analyses of randomised controlled trials. Bmj-Brit Med J. 2014;348. 

women 2015 [March 7, 2018]. Available from: https://www.gov.uk/government/publications/chief-medical-officer-annual-report-2014womens-health. economics make us healthier? Brit Med J. 2012;344. American people 2015 [07/03/2018]. Available from: https://obamawhitehouse.archives.gov/the-press-office/2015/09/15/executive-order-usingbehavioral-science-insights-better-serve-american . incentives for changing habitual health-related behaviors: A systematic review and metaanalysis. Prev Med. 2015;75:75-85.

10. Adams J, Giles EL, McColl E, Sniehotta FF. Carrots, sticks and health behaviours: A framework for documenting the complexity of financial incentive interventions to change health behaviours. Health Psychol Rev. 2014;8(3):286-95. Agricultural and Food Economics. 2013;1(1):19.

439 12. Reynolds B. A review of delay-discounting research with humans: relations to drug use and gambling. Behav Pharmacol. 2006;17(8):651-67. 

smoking: A systematic review and network analysis. Int J Epidemiol. 2017;46(3):869-.

443 14. Barlow P, Reeves A, McKee M, Galea G, Stuckler D. Unhealthy diets, obesity and time 444 discounting: A systematic literature review and network analysis. Obes Rev. 2016;17(9):810445 9. unhealthy behavior? A systematic review and reinforcement learning perspective. Front Behav Neurosci. 2014;8. Psychol Anim B. 1984;10(4):426-36. delay discounting in obesity. Physiology \& Behavior. 2016;162:46-51.

454 reconsideration. Eur Econ Rev. 2014;71:15-33. Learning; 2015.

457 20. Herzog R, Alvarez-Pasquin MJ, Diaz C, Del Barrio JL, Estrada JM, Gil A. Are healthcare 458 workers' intentions to vaccinate related to their knowledge, beliefs and attitudes? A 459 systematic review. Bmc Public Health. 2013;13. 
$479 \quad 2003 ; 11(2): 131-8$. 2009;151(4):264-W64.

21. Moher D, Liberati A, Tetzlaff J, Altman DG, Grp P. Preferred reporting items for systematic reviews and meta-Analyses: The PRISMA statement. Ann Intern Med.

22. Feda DM, Roemmich JN, Roberts A, Epstein LH. Food reinforcement and delay discounting in zBMI-discordant siblings. Appetite. 2015;85:185-9.

23. Reimers S, Maylor EA, Stewart N, Chater N. Associations between a one-shot delay discounting measure and age, income, education and real-world impulsive behavior. Pers Indiv Differ. 2009;47(8):973-8.

24. Golsteyn BHH, Gronqvist H, Lindahl L. Adolescent time preferences predict lifetime outcomes. Econ J. 2014;124(580):F739-F61.

25. Budria S, Lacomba JA, Lagos F, Swedberg P. When obese people are more patient than non-obese people a study of post-surgery individuals in a weight loss association. Rev Int Sociol. 2012;70:83-98.

26. Chabris CF, Laibson D, Morris CL, Schuldt JP, Taubinsky D. Individual laboratorymeasured discount rates predict field behavior. J Risk Uncertainty. 2008;37(2-3):237-69.

27. Conell-Price L, Jamison J. Predicting health behaviors with economic preferences \& locus of control. J Behav Exp Econ. 2015;54:1-9.

28. Epstein LH, Richards JB, Saad FG, Paluch RA, Roemmich JN, Lerman C. Comparison between two measures of delay discounting in smokers. Exp Clin Psychopharm. 
29. Fields SA, Sabet M, Peal A, Reynolds B. Relationship between weight status and delay discounting in a sample of adolescent cigarette smokers. Behav Pharmacol. 2011;22(3):266-8.

30. Fields SA, Sabet M, Reynolds B. Dimensions of impulsive behavior in obese, overweight, and healthy-weight adolescents. Appetite. 2013;70:60-6.

31. Kulendran M, Vlaev I, Sugden C, King D, Ashrafian H, Gately P, et al. Neuropsychological assessment as a predictor of weight loss in obese adolescents. Int J Obesity. 2014;38(4):507-12.

32. Richards TJ, Hamilton SF. Obesity and hyperbolic discounting: An experimental analysis. J Agr Resour Econ. 2012;37(2):181-98.

33. Rieger M. Risk aversion, time preference and health production: Theory and empirical evidence from Cambodia. Econ Hum Biol. 2015;17:1-15.

34. Simmank J, Murawski C, Bode S, Horstmann A. Incidental rewarding cues influence eeconomic decisions in people with obesity. Front Behav Neurosci. 2015;9.

35. Sutter M, Kocher MG, Glatzle-Rutzler D, Trautmann ST. Impatience and uncertainty: Experimental decisions predict adolescents' field behavior. Am Econ Rev. 2013;103(1):51031.

36. Sabet M. Impulsivity and obesity in adolescents: The Ohio State University; 2011.

37. de Oliveira ACM, Leonard TCM, Shuval K, Skinner CS, Eckel C, Murdoch JC. Economic preferences and obesity among a low-income African American community. Journal of Economic Behavior \& Organization. 2016;131:196-208. 
501

502

503

504

505

506

507

508

509

510

511

512

513

514

38. Rustichini A, DeYoung CG, Anderson JE, Burks SV. Toward the integration of personality theory and decision theory in explaining economic behavior: An experimental investigation. J Behav Exp Econ. 2016;64:122-37.

39. Hendrickson KL, Rasmussen EB. Effects of mindful eating training on delay and probability discounting for food and money in obese and healthy-weight individuals. Behav Res Ther. 2013;51(7):399-409.

40. Hendrickson KL, Rasmussen EB, Lawyer SR. Measurement and validation of measures for impulsive food choice across obese and healthy-weight individuals. Appetite. 2015;90:254-63.

41. Booij AS, van Praag BMS. A simultaneous approach to the estimation of risk aversion and the subjective time discount rate. Journal of Economic Behavior \& Organization. 2009;70(1-2):374-88.

42. Adams J, Nettle D. Time perspective, personality and smoking, body mass, and physical activity: An empirical study. Brit J Health Psych. 2009;14:83-105.

43. Bickel WK, Wilson AG, Franck CT, Mueller ET, Jarmolowicz DP, Koffarnus MN, et al. Using crowdsourcing to compare temporal, social temporal, and probability discounting among obese and non-obese individuals. Appetite. 2014;75:82-9.

44. Dassen FCM, Houben K, Jansen A. Time orientation and eating behavior: Unhealthy eaters consider immediate consequences, while healthy eaters focus on future health. Appetite. 2015;91:13-9. 

and behavioral measures of impulsivity. Appetite. 2015;95:375-82.

523

524

46. Nederkoorn C, Smulders FTY, Havermans RC, Roefs A, Jansen A. Impulsivity in obese women. Appetite. 2006;47(2):253-6.

47. Yeomans MR, Leitch M, Mobini S. Impulsivity is associated with the disinhibition but not restraint factor from the Three Factor Eating Questionnaire. Appetite. 2008;50(2-3):46976.

48. Verdejo-Garcia A, Perez-Exposito M, Schmidt-Rio-Valle J, Fernandez-Serrano MJ, Cruz F, Perez-Garcia M, et al. Selective alterations within executive functions in adolescents with excess weight. Obesity. 2010;18(8):1572-8.

49. Chan WS. Delay discounting and response disinhibition moderate associations between actigraphically measured sleep parameters and body mass index. Journal of Sleep Research. 2017;26(1):21-9.

50. Hendrickson KL, Rasmussen EB. Mindful eating reduces impulsive food choice in adolescents and adults. Health Psychology. 2017;36(3):226-35.

51. VanderBroek-Stice L, Stojek MK, Beach SRH, VanDellen MR, MacKillop J.

Multidimensional assessment of impulsivity in relation to obesity and food addiction. Appetite. 2017;112:59-68.

52. Jarmolowicz DP, Cherry JBC, Reed DD, Bruce JM, Crespi JM, Lusk JL, et al. Robust relation between temporal discounting rates and body mass. Appetite. 2014;78:63-7. 
$54153 . \quad$ Courtemanche C, Heutel G, McAlvanah P. Impatience, incentives and obesity. Econ J. $542 \quad 2015 ; 125(582): 1-31$.

543 54. Kang Ml, Ikeda S. Time discounting, present biases, and health-related behaviors:

544 Evidence from Japan. Econ Hum Biol. 2016;21:122-36.

545 55. Appelhans BM, Woolf K, Pagoto SL, Schneider KL, Whited MC, Liebman R. Inhibiting 546 food reward: Delay discounting, food reward sensitivity, and palatable food intake in 547 overweight and obese women. Obesity. 2011;19(11):2175-82.

548 56. Appelhans BM, Waring ME, Schneider KL, Pagoto SL, DeBiasse MA, Whited MC, et al. 549 Delay discounting and intake of ready-to-eat and away-from-home foods in overweight and 550 obese women. Appetite. 2012;59(2):576-84.

551 57. Bongers P, van de Giessen E, Roefs A, Nederkoorn C, Booij J, van den Brink W, et al.

552 Being impulsive and obese increases susceptibility to speeded detection of high-calorie 553 foods. Health Psychology. 2015;34(6):677-85.

554 58. Buono FDW, Seth W.; Sprong, Matthew E. Decision-making deficient in overweight 555 and obese adolescents: Evidence from door open and delay discounting tasks. Behavior 556 Analysis: Research and Practice. 2015;15(2):9.

557 59. Chen GC, Tai-sheng; Li, Zhi-hua; Liu, Wen-li. Decision-making deficient in overweight 558 and obese adolescents: Evidence from door open and delay discounting tasks. Chinese 559 Journal of Clinical Psychology. 2015;23(1):10. 

al. Insulin, central dopamine D2 receptors, and monetary reward discounting in obesity. Plos One. 2015;10(7).

61. Epstein LH, Jankowiak N, Fletcher KD, Carr KA, Nederkoorn C, Raynor HA, et al. Women who are motivated to eat and discount the future are more obese. Obesity. 2014;22(6):1394-9.

62. Garza KB, Harris CV, Bolding MS. Examination of value of the future and health beliefs to explain dietary and physical activity behaviors. Res Soc Admin Pharm. 2013;9(6):851-62.

63. Garza KB, Ding M, Owensby JK, Zizza CA. Impulsivity and fast-food consumption: A cross-sectional study among working adults. Journal of the Academy of Nutrition and Dietetics. 2016;116(1):61-8. Med. 2014;108:74-80.

574 65. Lu QY, Tao FB, Hou FL, Zhang ZC, Sun Y, Xu YY, et al. Cortisol reactivity, delay

575 discounting and percent body fat in Chinese urban young adolescents. Appetite. 2014;72:13-

57620.

577 66. Manwaring JL, Green L, Myerson J, Strube MJ, Wilfley DE. Discounting of various

578 types of rewards by women with and without binge eating disorder: Evidence for general 579 rather than specific differences. Psychol Rec. 2011;61(4):561-82. 

probability discounting for food in humans. Behav Process. 2010;83(1):23-30.

68. Weller RE, Cook EW, Avsar KB, Cox JE. Obese women show greater delay discounting than healthy-weight women. Appetite. 2008;51(3):563-9.

69. Thamotharan S, Lange K, Ramos A, Fields S. Examining weight concern and delay discounting in adolescent females. Eating Behaviors. 2016;21:228-31.

70. Borghans L, Golsteyn BHH. Time discounting and the body mass index: Evidence from the Netherlands. Econ Hum Biol. 2006;4(1):39-61.

71. Davis C, Patte K, Curtis C, Reid C. Immediate pleasures and future consequences. A neuropsychological study of binge eating and obesity. Appetite. 2010;54(1):208-13.

72. Dodd MC. Intertemporal discounting as a risk factor for high BMI: Evidence from Australia, 2008. Econ Hum Biol. 2014;12:83-97.

73. Ikeda S, Kang MI, Ohtake F. Hyperbolic discounting, the sign effect, and the body mass index. J Health Econ. 2010;29(2):268-84.

74. Tu Q, Donkers B, Melenberg B, van Soest A. The time preference of gains and losses 2004 [March 7, 2018]. Available from:

https://www.researchgate.net/publication/265893119 The Time Preference of Gains an

d Losses.

75. Schiff S, Amodio P, Testa G, Nardi M, Montagnese S, Caregaro L, et al. Impulsivity toward food reward is related to BMI: Evidence from intertemporal choice in obese and normal-weight individuals. Brain and Cognition. 2016;110:112-9. 
601

602

603

604

605

606

607

608

609

610

611

612

613

614

615

616

617
76. Takagi D, Kondo N, Takada M, Hashimoto H. Educational attainment, time preference, and health-related behaviors: A mediation analysis from the J-SHINE survey. Soc Sci Med. 2016;153:116-22.

77. Wang M, Rieger MO, Hens T. How time preferences differ: Evidence from 53 countries. Journal of Economic Psychology. 2016;52:115-35.

78. Bickel WK, Moody L, Higgins ST. Some current dimensions of the behavioral economics of health-related behavior change. Prev Med. 2016;92:16-23.

79. Bickel WK, Miller ML, Yi R, Kowal BP, Lindquist DM, Pitcock JA. Behavioral and neuroeconomics of drug addiction: Competing neural systems and temporal discounting processes. Drug Alcohol Depen. 2007;90:S85-S91.

80. Koffarnus MN, Jarmolowicz DP, Mueller ET, Bickel WK. Changing delay discounting in the light of the competing neurobehavioral decision systems theory: A review. J Exp Anal Behav. 2013;99(1):32-57.

81. Matjasko JL, Cawley JH, Baker-Goering MM, Yokum DV. Applying behavioral economics to public health policy illustrative examples and promising directions. Am J Prev Med. 2016;50(5):S13-S9. 